\title{
DISCOVERY OF THE HOST CLUSTER FOR THE FUNDAMENTAL CEPHEID CALIBRATOR ZETA GEMINORUM
}

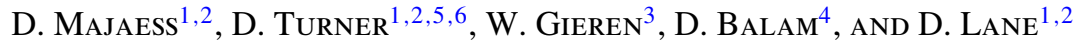 \\ ${ }^{1}$ Department of Astronomy \& Physics, Saint Mary's University, Halifax, NS, Canada \\ ${ }^{2}$ Abbey Ridge Observatory, Halifax, NS, Canada \\ ${ }^{3}$ Departamento de Astronomía, Universidad de Concepción, Concepción, Chile \\ ${ }^{4}$ Dominion Astrophysical Observatory, Victoria, BC, Canada \\ Received 2012 January 18; accepted 2012 February 16; published 2012 February 28
}

\begin{abstract}
New and existing CORAVEL, $U B V J H K_{s}, H S T$, HIP/Tycho, ARO, KPNO, and DAO observations imply that the fundamental Cepheid calibrator $\zeta$ Gem is a cluster member. The following parameters were inferred for $\zeta$ Gem from cluster membership and are tied to new spectral classifications (DAO) established for 26 nearby stars (e.g., HD53588/B7.5IV, HD54692/B9.5IV): $E_{B-V}=0.02 \pm 0.02, \log \tau=7.85 \pm 0.15$, and $d=355 \pm 15 \mathrm{pc}$. The mean distance to $\zeta$ Gem from cluster membership and six recent estimates (e.g., IRSB) is $d=363 \pm 9\left(\sigma_{\bar{x}}\right) \pm 26(\sigma)$ pc. The results presented here support the color-excess and HST parallax derived for the Cepheid by Benedict et al. Forthcoming precise proper motions (DASCH) and Chandra/XMM-Newton observations of the broader field may be employed to identify cluster members, bolster the cluster's existence, and provide stronger constraints on the Cepheid's fundamental parameters.
\end{abstract}

Key words: Hertzsprung-Russell and $\mathrm{C}-\mathrm{M}$ diagrams - open clusters and associations: general - stars: distances stars: variables: Cepheids

Online-only material: color figures

\section{INTRODUCTION}

An independent distance determination for $\zeta$ Gem is desirable since Hubble Space Telescope (HST) and HIP parallaxes for the classical Cepheid exhibit an unsatisfactory spread: $d=358-422$ pc (Perryman \& ESA 1997; van Leeuwen et al. 2007; Benedict et al. 2007). Establishing reliable parameters for $\zeta \mathrm{Gem}$ is particularly important given the Carnegie Hubble and $\mathrm{SH}_{0}$ ES projects (Macri \& Riess 2009; Freedman \& Madore 2010) are relying on HST calibrators (Benedict et al. 2007) to break degeneracies hindering the selection of a cosmological model (Riess et al. 2011). The Carnegie Hubble project shall likewise employ Galactic calibrators tied to open clusters (Turner 2010) featuring IRSB corroborated distances (Gieren et al. 2005; Storm et al. 2011). The classical Cepheid $\zeta$ Gem is the second longest period calibrator possessing a precise $H S T$ parallax (Benedict et al. 2002, 2007), and a solid calibration for such stars is needed since longer-period Cepheids may be less affected by (insidious) photometric contamination (Macri et al. 2006, their Figure 17). Moreover, longer-period Cepheids are detectable in distant galaxies owing to their increased luminosity relative to shorter-period Cepheids (e.g., Gieren et al. 2009, their Figure 1). Sampling remote galaxies in the Hubble flow mitigates uncertainties tied to peculiar velocity corrections and hence $H_{0}$ (Freedman et al. 2001, their Figure 1).

In this study, CORAVEL, UBVJHK,$H S T$, HIP/Tycho, $\mathrm{KPNO}$, and DAO spectroscopic observations are employed to identify stars potentially associated with $\zeta$ Gem, thereby permitting its fundamental properties to be deduced from cluster membership $\left(\log \tau, E_{B-V}\right.$, and $\left.W_{V I_{c}, 0}\right)$.

\footnotetext{
5 Visiting Astronomer, David Dunlap Observatory.

6 Visiting Astronomer, Kitt Peak National Observatory.
}

\section{ANALYSIS}

HIP/Tycho data were examined for stars surrounding $\zeta$ Gem which exhibit similar proper motions: $-10<\mu_{\alpha}<-2$ and $-7<\mu_{\delta}<2$ (Perryman \& ESA 1997; van Leeuwen et al. 2007). Stars which display proper motions that deviate significantly from $\zeta$ Gem were eliminated via the aforementioned limits. However, countless field stars likewise exhibit proper motions marginally offset from zero. The available proper motions could not be employed to substantiate the cluster's existence given their uncertainties (Majaess et al. 2012, see their Figure 3 for $\delta$ Cep). Stars redder than $B-V \sim 0.14$ were culled to further mitigate field contamination (e.g., red clump giants). $U B V J H K_{s}$ photometry was tabulated for the remaining sample using the compilations of Mermilliod (1991), Perryman \& ESA (1997), and Cutri et al. (2003).

\subsection{Reddening and Age}

An analysis of all stars near $\zeta$ Gem featuring $U B V$ photometry (Mermilliod 1991) confirms that the field is comparatively unreddened (Figure 1). The mean color excess inferred from new DAO spectra (Figure 2) for probable cluster members in Table 1 is $E(B-V)=0.019 \pm 0.017$. The findings support the reddening determined for $\zeta$ Gem by Benedict et al. (2007).

The cluster age can be constrained by examining the spectral type of members near the turnoff. A distinct sequence of B-stars is visible in the $B V J H$ color-magnitude diagrams (Figure 3, $r \leqslant 2.5$ from $\zeta$ Gem). The stars HD51102, HD51353, HD53588, and HD55919 aggregate near the turnoff and exhibit B6-B7 spectral classes (Table 1). For example, HD53588 displays $U B V$ colors and a $\mathrm{DAO}$ spectrum conducive to a $\mathrm{B} 7.5$ IV (Table 1). The star's radial velocity ( $\mathrm{RV}=10 \pm 3 \mathrm{~km} \mathrm{~s}^{-1}$ ) is consistent with that established for $\zeta \mathrm{Gem}\left(\mathrm{RV} \sim 7 \mathrm{~km} \mathrm{~s}^{-1}\right)$. However, cluster membership cannot be established solely on the basis of consistent radial velocities since the predicted 


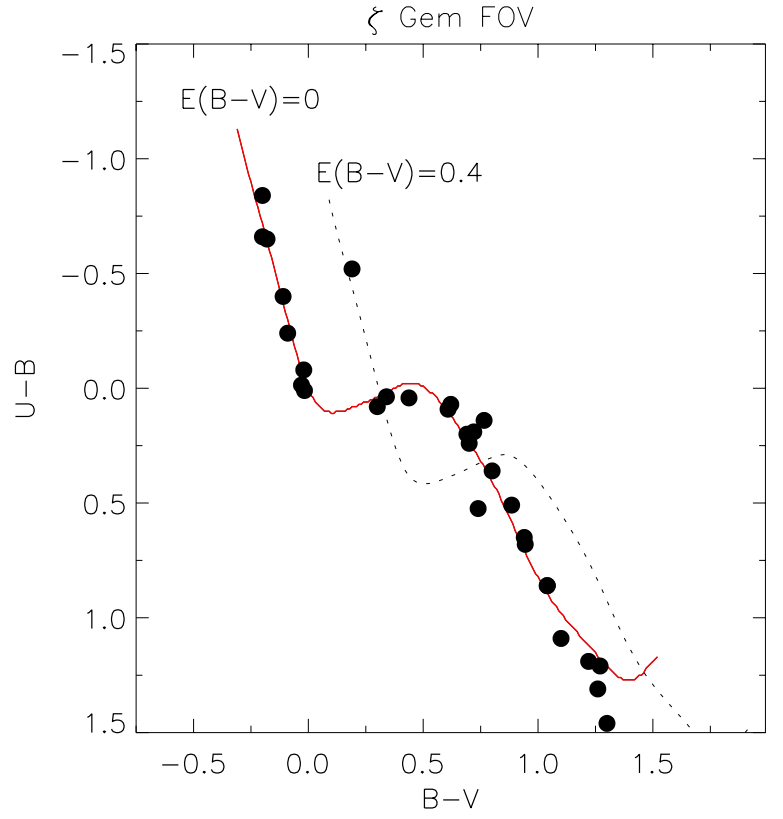

Figure 1. Color-color diagram for all stars $r \leqslant 4^{\circ}$ from $\zeta$ Gem which possess $U B V$ photometry (Mermilliod 1991). The field is comparatively unreddened. The intrinsic $U B V$ relation and reddening law for the region were adopted from Turner $(1976,1989)$. Most objects featured in the diagram are unassociated with $\zeta$ Gem.

(A color version of this figure is available in the online journal.)

radial-velocity-distance gradient along $\ell \sim 197^{\circ}$ is shallow. Membership for the two earliest type of stars examined is less certain (HD50767, HD51354; Table 1). A spectroscopic parallax for HD50767 implies that the star is well behind the cluster. The spectroscopic and HIP parallaxes for HD50767 disagree. The intrinsic colors for HD51354 $\left((B-V)_{0}:(U-B)_{0}=-0.18:-0.65\right)$ and the DAO spectrum indicate that the star is a B3 Vnne $(\mathrm{H} \beta$ emission). A mean of the HIP parallaxes established for HD51354 (Table 1) is consistent with that established for $\zeta$ Gem $(\pi=2.78 \pm 0.18$ mas; Benedict et al. 2007). The Perryman \& ESA (1997) parallax for HD51354 is $\pi=2.57 \pm 0.81$ mas, whereas the revised HIP parallax is $\pi=3.75 \pm 0.47$ mas (van Leeuwen et al. 2007). Spectroscopic parallaxes for emission stars (i.e., HD51354) are unreliable.

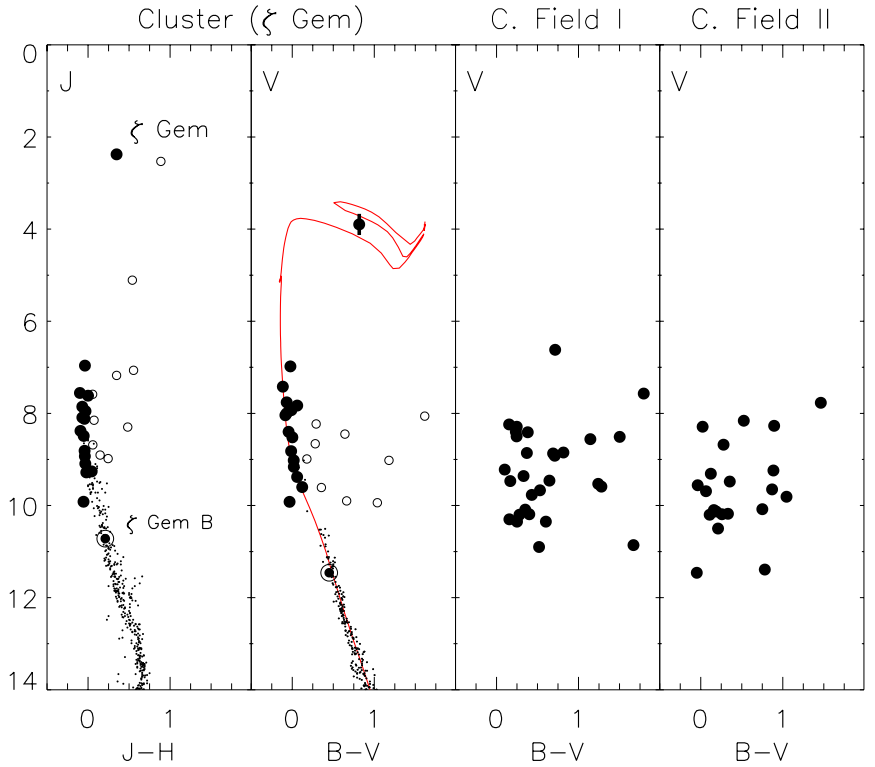

Figure 3. Left to right: $J H B V$ color-magnitude diagrams for HIP/Tycho stars within $r \leqslant 2.5$ of $\zeta$ Gem featuring $-10<\mu_{\alpha}<-2$ and $-7<\mu_{\delta}<2$. An early-type cluster sequence is absent from the comparison fields (panels 3 and 4, HIP/Tycho), which encompass $r \leqslant 4^{\circ}$. Small dots denote calibration stars from Majaess et al. (2011a), which were employed to tie the cluster distance to a geometrically anchored scale (van Leeuwen 2009; Majaess et al. 2011a). Open circles are likely field stars. Panel 2, a Padova $\log \tau=7.85$ isochrone was applied. The stars $\zeta$ Gem and $\zeta$ Gem B are the brightest cluster member (amplitude variation indicated) and circled dot accordingly.

(A color version of this figure is available in the online journal.)

A $\log \tau=7.85 \pm 0.15$ Padova isochrone ${ }^{7}$ provides the best match to the data (Figure 3). The age agrees with that predicted for stars aggregating near the turnoff and the age inferred for $\zeta$ Gem from its pulsation period (Turner 1996; Bono et al. 2005).

\subsection{Lower-mass Cluster Members}

Turner \& Fernie (1978) noted that $\zeta$ Gem B (J2000 07:04:12.73 +20:34:21.3) may be associated with $\zeta$ Gem. The

7 http://pleiadi.pd.astro.it/

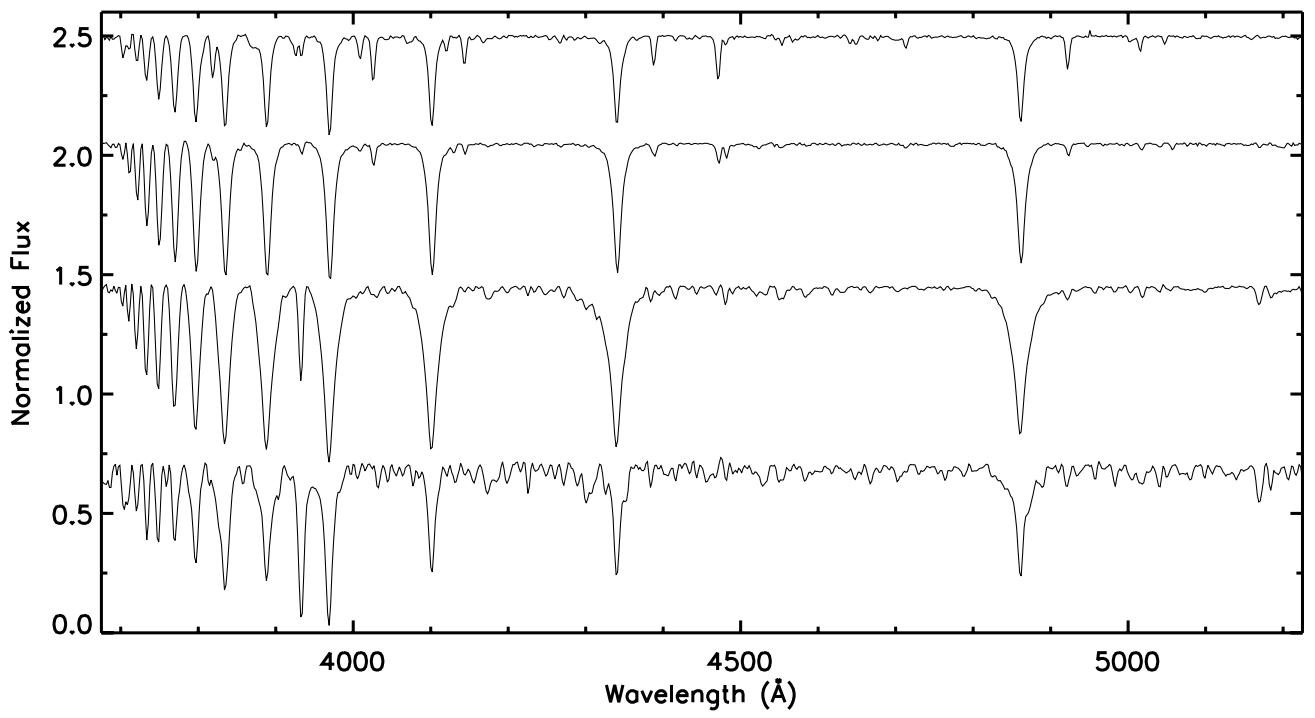

Figure 2. Subset of the new DAO spectra obtained for objects in the field of the classical Cepheid $\zeta$ Gem. From top to bottom the spectra correspond to HD50767, HD53588, HD52474, $\zeta$ Gem B. 
Table 1

Stars Near $\zeta$ Gem

\begin{tabular}{|c|c|c|c|c|c|c|c|c|c|}
\hline ID & $V$ & $B-V$ & $U-B$ & $J$ & $H$ & $K_{s}$ & $\mathrm{SpT}^{\mathrm{a}}$ & $\pi(\mathrm{P} 97)^{\mathrm{b}}$ & $\pi(\mathrm{V} 07)^{\mathrm{b}}$ \\
\hline$\zeta \mathrm{Gem}^{\mathrm{c}}$ & 3.90 & 0.82 & $\ldots$ & 2.38 & 2.03 & 2.14 & . & $2.79 \pm 0.81$ & $2.37 \pm 0.30$ \\
\hline HD 49381 & 6.80 & 0.01 & $\ldots$ & 6.66 & 6.70 & 6.65 & A0III & $3.87 \pm 0.96$ & $3.12 \pm 0.47$ \\
\hline HD 50634 & 6.98 & -0.02 & $\ldots$ & 6.96 & 7.00 & 6.96 & B9.5II & $2.41 \pm 0.98$ & $3.91 \pm 0.60$ \\
\hline HD 51354 & 7.12 & -0.18 & -0.65 & 7.20 & 7.18 & 7.05 & B3Vnne & $2.57 \pm 0.81$ & $3.75 \pm 0.47$ \\
\hline HD 53588 & 7.20 & -0.11 & -0.40 & 7.33 & 7.44 & 7.44 & B7.5IV & $4.67 \pm 0.87$ & $3.96 \pm 0.56$ \\
\hline HD 51102 & 7.42 & -0.12 & $\ldots$ & 7.56 & 7.66 & 7.70 & B6V & $0.36 \pm 1.00$ & $1.59 \pm 0.52$ \\
\hline HD 55919 & 7.43 & -0.09 & $\ldots$ & 7.60 & 7.68 & 7.72 & B7IV-V & $3.98 \pm 0.79$ & $3.86 \pm 0.60$ \\
\hline HD 52372 & 7.58 & 0.03 & $\ldots$ & 7.42 & 7.45 & 7.43 & A1III & $1.94 \pm 0.93$ & $2.17 \pm 0.86$ \\
\hline HD 50767 & 7.70 & -0.20 & -0.84 & 8.09 & 8.20 & 8.25 & $\mathrm{~B} 2 \mathrm{~V}$ & $2.15 \pm 1.01$ & $2.52 \pm 0.74$ \\
\hline HD 51353 & 7.76 & -0.07 & $\ldots$ & 7.86 & 7.93 & 7.92 & B7V & $2.00 \pm 0.94$ & $3.22 \pm 0.64$ \\
\hline HD 52474 & 7.83 & 0.06 & $\ldots$ & 7.62 & 7.62 & 7.59 & $\mathrm{~A} 2 \mathrm{~V}$ & $3.07 \pm 1.02$ & $2.79 \pm 0.66$ \\
\hline HD 52422 & 7.93 & -0.01 & $\ldots$ & 7.95 & 7.98 & 7.97 & B9IV & $2.03 \pm 1.10$ & $3.65 \pm 0.86$ \\
\hline HD 52371 & 8.00 & -0.07 & $\ldots$ & 8.12 & 8.16 & 8.23 & B7V: & $1.43 \pm 0.97$ & $1.13 \pm 0.83$ \\
\hline HD 50509 & 8.04 & -0.08 & $\ldots$ & 8.09 & 8.16 & 8.16 & B8V & $-1.28 \pm 1.06$ & $0.88 \pm 0.72$ \\
\hline HD 54404 & 8.40 & -0.05 & $\ldots$ & 8.38 & 8.47 & 8.45 & B9IV & $3.12 \pm 1.05$ & $1.49 \pm 0.86$ \\
\hline HD 54692 & 8.52 & 0.00 & $\ldots$ & 8.49 & 8.54 & 8.53 & B9.5IV & $3.29 \pm 1.12$ & $2.26 \pm 0.84$ \\
\hline HD 50107 & 8.84 & -0.01 & $\ldots$ & 8.83 & 8.80 & 8.78 & $\ldots$ & $\ldots$ & $\ldots$ \\
\hline HD 53230 & 8.84 & 0.09 & $\ldots$ & 8.59 & 8.54 & 8.51 & $\ldots$ & $1.92 \pm 1.24$ & $2.56 \pm 1.02$ \\
\hline HD 53288 & 8.84 & -0.02 & -0.08 & 8.81 & 8.85 & 8.84 & B9.5V & $2.29 \pm 1.05$ & $1.53 \pm 0.83$ \\
\hline HD 50164 & 8.94 & -0.07 & $\ldots$ & 8.91 & 8.96 & 8.92 & B9Vnn & $3.82 \pm 1.33$ & $3.34 \pm 1.19$ \\
\hline HD 49824 & 8.97 & 0.10 & $\ldots$ & 8.70 & 8.66 & 8.67 & $\ldots$ & $0.33 \pm 1.25$ & $-0.74 \pm 0.89$ \\
\hline HD 263791 & 8.98 & -0.05 & $\ldots$ & 8.99 & 9.12 & 9.06 & B9V & $0.38 \pm 1.36$ & $0.17 \pm 1.24$ \\
\hline HD 51187 & 9.02 & 0.02 & $\ldots$ & 8.93 & 8.97 & 8.92 & $\mathrm{~A} 0 \mathrm{~V}$ & $\ldots$ & $\ldots$ \\
\hline HD 53473 & 9.16 & 0.02 & $\ldots$ & 9.09 & 9.13 & 9.07 & $\mathrm{~A} 0 \mathrm{~V}$ & $0.92 \pm 1.18$ & $0.30 \pm 0.99$ \\
\hline HD 51971 & 9.38 & 0.06 & $\ldots$ & 9.28 & 9.30 & 9.22 & A1V & $\ldots$ & $\ldots$ \\
\hline TYC 1352-582-1 & 9.60 & 0.12 & $\ldots$ & 9.26 & 9.22 & 9.15 & $\ldots$ & $\ldots$ & $\ldots$ \\
\hline $\mathrm{BD}+18^{\circ} 1470$ & 9.92 & -0.03 & $\ldots$ & 9.92 & 9.97 & 9.98 & B9.5V V $\mathrm{pr}-\mathrm{Eu}$ & $\ldots$ & $\ldots$ \\
\hline$\zeta \mathrm{Gem} \mathrm{B}^{\mathrm{d}}$ & 11.47 & 0.42 : & $\ldots$ & 10.72 & 10.51 & 10.46 & $\mathrm{~F} 4 \mathrm{~V}$ & $\ldots$ & $\ldots$ \\
\hline $07: 03: 23.1+20: 37: 59.5$ & 11.71 & $0.38:$ & $\cdots$ & 11.06 & 10.87 & 10.85 & $\ldots$ & $\cdots$ & $\cdots$ \\
\hline $07: 03: 52.6+20: 35: 16.3$ & 12.34 & $0.54:$ & $\ldots$ & 11.37 & 11.11 & 11.06 & F7V & $\ldots$ & $\ldots$ \\
\hline $07: 04: 28.6+20: 34: 47.3$ & 12.37 & 0.53: & $\ldots$ & 11.46 & 11.24 & 11.17 & $\ldots$ & $\ldots$ & $\ldots$ \\
\hline $07: 04: 40.4+20: 35: 13.1$ & 12.45 & $0.46:$ & $\ldots$ & 11.66 & 11.51 & 11.47 & $\ldots$ & $\ldots$ & $\ldots$ \\
\hline
\end{tabular}

Notes.

a Stars classified by D. Turner (DAO spectra).

b Perryman \& ESA (1997, P97), van Leeuwen (2007, V07).

${ }^{c}$ van Leeuwen et al. (2007) cite $\pi=2.71 \pm 0.17$ mas for $\zeta$ Gem.

${ }^{\mathrm{d}}$ The star $\zeta$ Gem B is a spectroscopic binary (Section 2.2). Benedict et al. (2007) classified $\zeta$ Gem B as an F3.5V.

stars are separated by $r \sim 1$ '.4. A mean radial velocity was determined for $\zeta$ Gem B from six CORAVEL measurements obtained at l'Observatoire de Haut-Provence (OHP). ${ }^{8}$ The radial velocities acquired from the OHP span -16.2 to $36.9 \mathrm{~km} \mathrm{~s}^{-1}$, yielding a mean of $9.9 \mathrm{~km} \mathrm{~s}^{-1} . \zeta \mathrm{Gem} \mathrm{B}$ is thus a spectroscopic binary since the uncertainty tied to an individual measurement is $1.4 \mathrm{~km} \mathrm{~s}^{-1}$. The mean radial velocity matches that established for the Cepheid to within the uncertainties. However, as noted in Section 2.1, the shallow radial-velocity-distance gradient along the line of sight requires that cluster membership be secured by independent means. $U B V$ photometry by Fernie (1969) indicates that $\zeta$ Gem B is an F-type star (Turner \& Fernie 1978). That is corroborated by the 2MASS colors for the object $((J-H):(H-K)=0.206: 0.056)$, which are indicative of an unreddened F5-F8V (Straižys \& Lazauskaitė 2009). A spectrogram of the star was acquired on HJD $=2,444,122.995$ from the $2.1 \mathrm{~m}$ telescope at Kitt Peak. That spectrogram and a DAO spectrum confirm that $\zeta$ Gem B is an F4V (Table 1, Figure 2).

\footnotetext{
8 M. Mayor kindly obtained CORAVEL observations of $\zeta$ Gem B for D. Turner.
}

The result is supported by Benedict et al. (2007), who classified the star as an F3.5V. The spectral and luminosity class for $\zeta$ Gem B is consistent with that expected for a cluster member at the star's location in the $B V J H$ color-magnitude diagrams (Figure 3).

DAO spectra were obtained for two additional stars in close proximity to $\zeta$ Gem $\left(r<6^{\prime}\right)$. The spectral and luminosity class for 2MASS $07041267+2030196$ is consistent with that expected for a cluster member at the star's location in the $B V J H$ color-magnitude diagrams (Figure 3, Table 1). The same is true for 2MASS 07035262+2035162. Benedict et al. (2007) inferred an analogous classification for 2MASS $07035262+2035162$ (F6V). Four additional stars in close proximity to $\zeta$ Gem exhibit multiband photometry conducive to late-type (potential) cluster members. New $B V$ observations for those stars were acquired from the Abbey Ridge Observatory (ARO; Lane 2008; Majaess et al. 2008). The data were processed via ARAP (Lane 2008) and DAOPHOT (Stetson 1987), and subsequently standardized to photometry obtained from the New Mexico State University $1 \mathrm{~m}$ telescope (T. Harrison 2012, private communication; see also Benedict et al. 2007). The following equations were derived 
to place the instrumental ARO photometry onto the Johnson system:

$$
\begin{aligned}
B-V & =(0.99 \pm 0.06) \times(b-v)-(0.57 \pm 0.08) \\
V-v & =-0.86 \pm 0.02
\end{aligned}
$$

Observations from the AAVSO's Bright Star Monitor (BSM) provided additional data for $\zeta$ Gem $B$ and 2MASS 07041267+2030196. The BSM is located at the Astrokolkhoz telescope facility near Cloudcroft, New Mexico.

HST WFC3 images (HST Proposal 12215, Evans 2009) reveal a star 15" west of the Cepheid at J2000 07:04:05.5 +20:34:12.0. The object was identified after subtracting (normalized) the image featuring $\zeta$ Gem from a master, which was constructed (median combine) using Cepheids observed for proposal 12215. The object displays a signal-to-noise-ratio greater than 20 in both WFC3 images. However, N. Evans (2012, private communication) noted that the star's photometry is not conducive to cluster membership.

Later-type stars associated with $\zeta$ Gem should presumably exhibit X-ray emission (Evans 2011). Additional observations are required to assess the candidates (e.g., Chandra/XMMNewton). The objects are not featured in the ROSAT catalogs.

\subsection{Cluster Distance}

A precise cluster distance may be determined since the reddening, age, and chemical composition of the sample are constrained $\left([\mathrm{Fe} / \mathrm{H}]_{\zeta \mathrm{Gem}} \sim 0\right.$; Luck et al. 2011). The magnitude shift required to overlay the intrinsic relation upon the data yields $d=355 \pm 15 \mathrm{pc}$ (Figure 3 ). The zero point is tied to seven benchmark open clusters $(d<250 \mathrm{pc})$ that exhibit matching $J H K_{s}$ and revised HIP distances (e.g., the Hyades, $\alpha$ Per, Coma Ber; van Leeuwen 2009; Majaess et al. 2011a). A redetermination of the HST parallax for the Hyades supports that scale (McArthur et al. 2011). The scale employed here is anchored to clusters where consensus exists, rather than to the discrepant case (i.e., the Pleiades). A ratio of total to selective extinction $R_{J}$ was adopted from Majaess et al. (2011b; see also Bonatto et al. 2004), whereas a value for $R_{V}$ was adopted from Turner (1976). Deviations from the canonical reddening law are mitigated since the field is comparatively unreddened (Figure 1, note that $V_{0}=V-E_{B-V} \times R_{V}$ ).

A mean distance inferred from potential members possessing revised HIP parallaxes is $d=366 \pm 57\left(\sigma_{\bar{x}}\right) \pm 196(\sigma)$ pc. Certain stars were excluded from the derivation since they exhibit uncertainties greater than the parallax cited, deviate significantly from the mean, or feature negative parallaxes (e.g., HD49824, $\pi=-0.74 \pm 0.89$ mas). Stars with spectral types inconsistent with cluster membership were likewise removed (e.g., HD50767, Section 2.1).

Three HIP parallaxes exist for $\zeta$ Gem: $\pi=2.79 \pm 0.81$ : $2.37 \pm 0.30: 2.71 \pm 0.17$ mas (Perryman \& ESA 1997; van Leeuwen 2007; van Leeuwen et al. 2007). The results inferred from cluster membership and $H S T$ observations for $\zeta$ Gem $(\pi=2.78 \pm 0.18$ mas; Benedict et al. 2007) support the van Leeuwen et al. (2007) HIP determination. Distances to $\zeta$ Gem are also available from the infrared surface brightness technique $(d=386 \pm 9$ pc; Storm et al. 2011) and the Palomar Testbed Interferometer (PTI; $d=336 \pm 44$ pc; Lane et al. 2000, and references therein). A straight mean of the six (+2 cluster based) estimates to $\zeta$ Gem yields $d=363 \pm 9\left(\sigma_{\bar{x}}\right) \pm 26(\sigma)$ pc. A weighted mean of $d=368$ pc was obtained by assigning $w=1$ for the HIP and PTI estimates, and $w=2$ for the rest.

\section{CONCLUSION AND FUTURE RESEARCH}

The evidence indicates that the $10^{\mathrm{d}}$ classical Cepheid calibrator $\zeta$ Gem belongs to a newly identified cluster (Figure 3). Potential members were identified on the basis of new and existing CORAVEL, UBVJHK,$H S T$, HIP/Tycho, KPNO, and DAO $(n=26)$ spectroscopic observations. The analysis indicates that the field is comparatively unreddened (Figure 1), and that $\zeta$ Gem exhibits $E(B-V)=0.019 \pm 0.017$ (Section 2.1). The age and distance were inferred from spectroscopic and multiband $U B V J H K_{S}$ analyses of the cluster stars, yielding $\log \tau=7.85 \pm 0.15$ and $d=355 \pm 15 \mathrm{pc}$ (Figure 3 ). The results support the parameters derived for $\zeta$ Gem by Benedict et al. (2007). However, the breadth of the standard deviation tied to several recent estimates for $\zeta$ Gem is unsatisfactory (Section 2.3, $d=363 \pm 9\left(\sigma_{\bar{x}}\right) \pm 26(\sigma)$ ), and continued research is required. CORAVEL, KPNO, and DAO observations indicate that $\zeta$ Gem B is an F-type spectroscopic binary. DAO spectra were likewise obtained for two other low-mass members in close proximity to $\zeta$ Gem. Additional observations are required to highlight bona fide members on the candidate list (Table 1). Those stars are potential members pending further evidence. Membership identification may be facilitated by obtaining precise proper motions for fainter stars near $\zeta$ Gem from photographic plates stored at the CfA (Grindlay 2007; DASCH). ${ }^{9}$ The plates offer multi-epoch coverage spanning a $\sim 100$ year baseline, and uncertainties are further mitigated owing to sizable statistics $\left(\sim(5-10) \times 10^{2}\right.$ plates per object $)$. Searching for X-ray emission from lower-mass stars near $\zeta$ Gem is likewise a viable pursuit for corroborating membership (Evans 2011; Evans et al. 2011). However, the star Polaris B is of a similar spectral type as $\zeta$ Gem B (Turner 1977) and does not exhibit X-ray emission (Evans et al. 2010).

At least two classical Cepheids featuring HST parallaxes are cluster members ( $\delta$ Cep and $\zeta$ Gem; de Zeeuw et al. 1999; Majaess et al. 2012). Cluster membership provides a means to secure independent fundamental parameters $\left(\log \tau, E_{B-V}\right.$, $W_{V I_{c}, 0}, \log L_{*} / L_{\odot}$, and $\left.M_{V}\right)$. The results shall complement a suite of diverse efforts unified by a common objective to reduce uncertainties associated with $H_{0}$ in order to constrain cosmological models (Feast 2008; Shappee \& Stanek 2011; Gerke et al. 2011; Ngeow 2011, 2012; Steer \& Madore 2011).

D.M. is grateful to the following individuals and consortia whose efforts fostered the research: F. van Leeuwen and M. Perryman (HIP), F. Benedict (HST), J.-C. Mermilliod, Skiff (2010), 2MASS, M. Mayor, N. Evans, T. Harrison, J. Rosvick, P. Stetson (DAOPHOT), WEBDA (Paunzen 2008), DAML (Dias et al. 2002), BSM/AAVSO (T. Krajci, A. Henden, M. Templeton, A. Price), CDS, arXiv, and NASA ADS. W.G. is grateful for support from the Chilean Center for Astrophysics FONDAP 15010003 and the BASAL Centro de Astrofisica y Tecnologias Afines (CATA) PFB-06/2007.

\section{REFERENCES}

Benedict, G. F., McArthur, B. E., Feast, M. W., et al. 2007, AJ, 133, 1810 Benedict, G. F., McArthur, B. E., Fredrick, L. W., et al. 2002, AJ, 124, 1695 Bonatto, C., Bica, E., \& Girardi, L. 2004, A\&A, 415, 571 Bono, G., Marconi, M., Cassisi, S., et al. 2005, ApJ, 621, 966 Cutri, R. M., Skrutskie, M. F., van Dyk, S., et al. 2003, The IRSA 2MASS All-Sky Point Source Catalog, NASA/IPAC Infrared Science Archive

\footnotetext{
9 Digital Access to a Sky Century @ Harvard (DASCH),
} http://hea-www.harvard.edu/DASCH/ 
de Zeeuw, P. T., Hoogerwerf, R., de Bruijne, J. H. J., Brown, A. G. A., \& Blaauw, A. 1999, AJ, 117, 354

Dias, W. S., Alessi, B. S., Moitinho, A., \& Lépine, J. R. D. 2002, A\&A, 389, 871

Evans, N. 2009, HST Proposal, 12215

Evans, N. R. 2011, in IAU Symp. 272, Active OB Stars: Structure, Evolution, Mass Loss, and Critical Limits, ed. C. Neiner et al. (Cambridge: Cambridge Univ. Press), 537

Evans, N. R., DeGioia-Eastwood, K., Gagné, M., et al. 2011, ApJS, 194, 13

Evans, N. R., Guinan, E., Engle, S., et al. 2010, AJ, 139, 1968

Feast, M. W. 2008, in Proc. First Middle East-Africa Regional IAU Meeting, ed. A. W. Alsabti, A. A. Hady, \& V. Bothmer (Cairo: Univ. Cairo Press), arXiv:0806.3019

Fernie, J. D. 1969, J. R. Astron. Soc. Can., 63, 133

Freedman, W. L., \& Madore, B. F. 2010, ARA\&A, 48, 673

Freedman, W. L., Madore, B. F., Gibson, B. K., et al. 2001, ApJ, 553, 47

Gerke, J. R., Kochanek, C. S., Prieto, J. L., Stanek, K. Z., \& Macri, L. M. 2011, ApJ, 743, 176

Gieren, W., Pietrzyński, G., Soszyński, I., et al. 2005, ApJ, 628, 695

Gieren, W., Pietrzyński, G., Soszyński, I., et al. 2009, ApJ, 700, 1141

Grindlay, J. E. 2007, in ASP Conf. Ser. 373, The Central Engine of Active Galactic Nuclei, ed. L. C. Ho \& J.-M. Wang (San Francisco, CA: ASP), 711

Lane, B. F., Kuchner, M. J., Boden, A. F., Creech-Eakman, M., \& Kulkarni, S. R. 2000, Nature, 407, 485

Lane, D. J. 2008, J. Am. Assoc. Var. Star Obs., 36, 143

Luck, R. E., Andrievsky, S. M., Kovtyukh, V. V., Gieren, W., \& Graczyk, D. 2011, AJ, 142, 51

Macri, L. M., \& Riess, A. G. 2009, in AIP Conf. Proc. 1170, Stellar Pulsation: Challenges for Theory and Observation, ed. J. A. Guzik \& P. A. Bradley (Melville, NY: AIP), 23

Macri, L. M., Stanek, K. Z., Bersier, D., Greenhill, L. J., \& Reid, M. J. 2006, ApJ, 652,1133

Majaess, D. J., Turner, D. G., \& Gieren, W. 2012, ApJ, 747, 145

Majaess, D. J., Turner, D. G., Lane, D. J., \& Moncrieff, K. E. 2008, J. Am. Assoc. Var. Star Obs., 36, 90
Majaess, D. J., Turner, D. G., Lane, D. J., \& Krajci, T. 2011a, J. Am. Assoc. Var. Star Obs., 39, 219

Majaess, D. J., Turner, D. G., Moni Bidin, C., et al. 2011b, ApJ, 741, L27

McArthur, B. E., Benedict, G. F., Harrison, T. E., \& van Altena, W. 2011, AJ, 141,172

Mermilliod, J.-C. 1991, Homogeneous Means in the UBV System, Vizier Catalog

Ngeow, C.-C. 2011, in ASP Conf. Ser., 9th Pacific Rim Conference on Stellar Astrophysics (PRCSA2011), ed. S. Qian (San Francisco, CA: ASP), in press (arXiv:1111.2094)

Ngeow, C.-C. 2012, ApJS, in press (arXiv:1202.0339)

Paunzen, E. 2008, Contrib. Astron. Obs. Skalnate Pleso, 38, 435

Perryman, M. A. C., \& ESA 1997, in The Hipparcos and Tycho Catalogues, Astrometric and Photometric Star Catalogues Derived from the ESA Hipparcos Space Astrometry Mission (ESA Special Publication, Vol. 1200; Noordwijk: ESA)

Riess, A. G., Macri, L., Casertano, S., et al. 2011, ApJ, 730, 119

Shappee, B. J., \& Stanek, K. Z. 2011, ApJ, 733, 124

Skiff, B. 2010, General Catalogue of Stellar Spectral Classifications, Vizier Catalog

Steer, I., \& Madore, B. 2011, NED-D: A Master List of Redshift-Independent Extragalactic Distance, http://ned.ipac.caltech.edu/Library/Distances/

Stetson, P. B. 1987, PASP, 99, 191

Storm, J., Gieren, W., Fouqué, P., et al. 2011, A\&A, 534, A94

Straižys, V., \& Lazauskaitė, R. 2009, Balt. Astron., 18, 19

Turner, D. G. 1976, AJ, 81, 1125

Turner, D. G. 1977, PASP, 89, 550

Turner, D. G. 1989, AJ, 98, 2300

Turner, D. G. 1996, J. R. Astron. Soc. Can., 90, 82

Turner, D. G. 2010, Ap\&SS, 326, 219

Turner, D. G., \& Fernie, J. D. 1978, Inf. Bull. Var. Stars, 1509, 1

van Leeuwen, F. 2007, A\&A, 474, 653

van Leeuwen, F. 2009, A\&A, 497, 209

van Leeuwen, F., Feast, M. W., Whitelock, P. A., \& Laney, C. D. 2007, MNRAS, 379,723 